Pacific Journal of Mathematic 


\title{
A MEAN VALUE THEOREM FOR QUADRATIC FIELDS
}

\author{
RAYMOND G. AYouB
}

1. Introduction. Let $K$ be an algebraic extension of the rationals of degree $k, F(n)$ denote the number of ideals whose norm is the rational integer $n, H(x)=\sum_{n \leq x} F(n)$. Let $\zeta(s, K)$ denote the Dedekind zeta function for the field $K$, that is,

$$
\zeta(s, K)=\sum_{\mathfrak{U}} \frac{1}{N(\mathfrak{H}) s}=\sum_{n=1}^{\infty} F(n)
$$

and $\alpha$ the residue of $\zeta(s, K)$ at its simple pole at $s=1$.

It has long been known [8] that

$$
H(x)=\alpha x+\Delta_{k}(x)
$$

where

$$
\Delta_{k}(x)=0\left(x^{1-1 / k}\right)
$$

and Landau [3] proved that

$$
\Delta_{k}(x)=0\left(x^{1-2 /(k+1)}\right)
$$

The precise nature of the error term $A_{k}(x)$ seems rather intractable and seems to be intimately related to the behavior of the function $\zeta(s, K)$ in the critical strip. Of considerable interest is the particular case when $K$ is the Gaussian field $R(i)$, for in that case $\Delta_{k}(x)$ is the error term in the classical problem of the number of lattice points in a circle.

Using some results of class field theory, Suetuna [4] has obtained an improvement of Landau's result in the case when the field is normal and has abelian Galois group and $k \geqq 4$. For, when the field is abelian, the theorems of Weber-Takagi tell us that $\zeta(s, K)$ is the product of $k$ Dirichlet $L$-functions belonging to primitive characters. Applying his approximate functional equation for the Dirichlet $L$-functions, and using refined estimates for these in the critical strip, Suetuna then obtains the desired result.

In the light of more recent techniques for dealing with the Riemann zeta function, further improvements are possible. The devices for handing the zeta function are used for the $L$-functions and the class

Received February 12, 1957. 
field theorems again applied. We omit details.

It is our object here to study the problem of a mean value for $\Delta_{k}(x)$. We are able to obtain a precise result but only for quadratic fields. Some known results follow as corollaries when the quadratic field is specified: for example when the field is $R(i)$.

We use as our tools a result of Suetuna [4] and a technique devised by Titchmarsh [5], [6] for the corresponding results for the closely allied problem of $\sum_{n \leq x} d_{k}(n)$ where $d_{k}(n)$ is the number of solutions of $n=n_{1} n_{2} \cdots n_{k}$. We follow closely Titchmarsh's method.

2. Notations and statement of Main theorem. Let $k=2, \Delta_{2}(x)=\Delta(x)$, $s=\sigma+i t$. Following Hardy [6], we define the mean value of $\Delta(x)$ as the least number $\beta$ such that

$$
\frac{1}{x} \int_{0}^{x} \Delta^{2}(t) d t=0\left(x^{2 \beta+\varepsilon}\right)
$$

It is our object to prove the following.

MaIn Theorem. $\beta=\frac{1}{4}$.

We first relate $\beta$ to the convergence of an integral.

THEOREM 2.1 Let $\gamma$ be the lower bound of positive numbers $\sigma$ for which

$$
I=\int_{-\infty}^{\infty} \frac{|\zeta(\sigma+i t, K)|^{2}}{|\sigma+i t|^{2}} d t
$$

converges. Then $\beta=\gamma$ and if $\sigma>\beta$, then

$$
2 \pi \int_{0}^{\infty} \Delta^{2}(x) x^{-2 \sigma-1} d x=I
$$

Proof. Using the classical formula for the sum of the coefficients of a Dirichlet series, we have,

$$
\begin{aligned}
H(x) & =\frac{1}{2 \pi i} \int_{c-i \infty}^{c+i \infty} \zeta(s, K) \\
s & x^{s} p s \\
& =\frac{1}{2 \pi i} \lim _{T \rightarrow \infty} \int_{c-i T}^{c+i T} \zeta(s, K) \\
s & x^{s} d s
\end{aligned}
$$

We move the line of integration to $\sigma=\delta$, where $0<\delta<1$. Using Cauchy's theorem and taking account of the residue at $s=1$, we get, if $\delta$ is chosen appropriately close to 1 , 


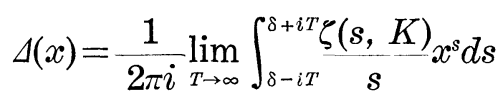

The bound on $\delta$ follows from the implied calculation but we do not need it since we now prove the validity of (3) for the range $r<\delta<1$. To do this, we note that by some general theorems of analysis [2], and taking account of $(1), \frac{\zeta(s, K)}{s}$ tends uniformly to 0 as $t \rightarrow \pm \infty$. With this established, we integrate around the rectangle defined by $\delta^{\prime}-i T$, $\delta-i T, \delta+i T, \delta^{\prime}+i T$ with $\gamma<\delta^{\prime}<\delta<1$, let $T \rightarrow \infty$, and deduce the desired result.

With Titchmarsh, we now use the theory of Mellin transforms. The Parseval theorem for the Mellin integral gives [7],

$$
\begin{aligned}
\frac{1}{2 \pi} \int_{-\infty}^{\infty} \frac{|\zeta(\delta+i t), k|^{2}}{|\delta+i t|^{2}} & =\int_{0}^{\infty} \Delta^{2}\left(\frac{1}{x}\right) x^{2 \delta-1} d x \\
& =\int_{0}^{\infty} \Delta^{2}(x) x^{-2 \delta-1} d x
\end{aligned}
$$

as long as $\gamma<\delta<1$. This implies that $\beta \leqq \gamma$ : by (4)

$$
\int_{\frac{x}{2}}^{x} A^{2}(x) x^{-2 \delta-1} d x<C(\delta)=C_{1}
$$

that is,

$$
\int_{\frac{x}{2}}^{x} \Delta^{2}(x) d x<C_{2} x^{2 \delta+1}
$$

Replacing $x$ by $x / 2, x / 4, x / 8, \cdots$ and adding, we deduce

$$
\int_{1}^{x} \Delta^{2}(x) d x<C_{3} x^{2 \delta+1}
$$

whence $\beta \leqq \delta$, that is, $\beta \leqq \delta$.

To prove the reverse inequality, we have by Plancherel's form of the inverse Mellin transform [7],

$$
\frac{\zeta(s, K)}{s}=\int_{0}^{\infty} \Delta(x) x^{-s-1} d x
$$

where the right hand integral exists in the mean square sense for $\gamma<\delta<1$. Actually the right hand side is uniformly convergent for the range $\beta^{\prime}<\sigma<\beta^{\prime \prime}$ where $\beta<\beta^{\prime}<\beta^{\prime \prime}<1$. For, using the Schwartz inequality, 


$$
\int_{\frac{x}{2}}^{x}|\Delta(x)| x^{-\sigma-1} d x \leqq\left(\int_{\frac{x}{2}}^{x} A^{2}(x) d x\right)^{1 / 2}\left(\int_{\frac{x}{2}}^{x} x^{-2 \sigma-2}\right)^{1 / 2}=O\left(x^{2 \beta+1+\varepsilon}\right)^{1 / 2}\left(x^{-2 \sigma-1}\right)^{1 / 2}=O\left(x^{\beta-\sigma+\varepsilon}\right)
$$

putting $x=2,4,8, \cdots$ and adding, we get

$$
\int_{1}^{\infty}|\Delta(x)| x^{-\sigma-1} d x<\infty
$$

By a similar argument,

$$
\int_{0}^{\infty} \Delta^{2}(x) x^{-2 \delta-1}
$$

converges for $\beta<\delta<1$. Now

$$
\int_{0}^{\infty} \Delta(x) x^{-s-1} d x
$$

is an analytic function for $\beta<\sigma<1$, and hence

$$
\frac{1}{2 \pi} \int_{-\infty}^{\infty} \frac{|\zeta(\delta+i t, K)|^{2}}{|\delta+i t|^{2}} d t<\infty
$$

for $\beta<\delta<1$, whence $\gamma \leqq \delta$ that is, $\gamma \leqq \beta$ and the theorem is proved.

So far we have not made use of the condition $k=2$. Indeed Titchmarsh's method applies in quite a general setting. We require the condition however in the proof of the main theorem.

Lemma (Suetuna). If $\sigma>\frac{1}{2}$, then

$$
\lim _{T \rightarrow \infty} \frac{1}{2 T} \int_{-T}^{T}|\zeta(s, K)|^{2} d t=\sum_{n=1}^{\infty} \frac{F^{2}(n)}{n^{2 \sigma}}
$$

Proof of the Main Theorem. We first prove that $\beta \geqq \frac{1}{4}$. By the above lemma, we have for $\frac{1}{2}<\sigma<1$,

$$
\int_{\frac{T}{2}}^{T}|\zeta(s, K)|^{2}<C T
$$

Therefore for $0<\sigma<\frac{1}{2}, T>1$ and using Hecke's functional equation for $\zeta(s, K)$, (see for example. Landau [3]), we get

$$
\int_{-\infty}^{\infty} \frac{|\zeta(\sigma+i t, K)|^{2}}{|\sigma+i t|^{2}} d t>\int_{T^{-}}^{T} \frac{|\zeta(\sigma+i t, K)|^{2}}{|\sigma+i t|^{2}} d t>\frac{C_{1}}{T^{2}} \int_{\frac{T}{2}}^{T}|\zeta(\sigma+i t, K)|^{2} d t
$$




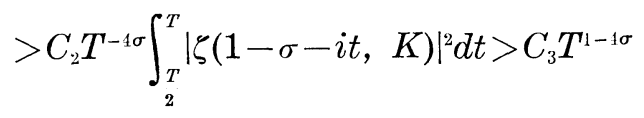

The right hand side tends to infinity if $\sigma<\frac{1}{4}$, whence $\beta \geqq \frac{1}{4}$.

Again by the above lemma, for $\frac{1}{2}<\sigma<1$

$$
\int_{1}^{T}|\zeta(\sigma+i t, K)|^{2} d t=O(T)
$$

Using the functional equation, we get for $0<\sigma<\frac{1}{2}$

$$
\begin{aligned}
\int_{1}^{T}|\zeta(\sigma+i t, K)|^{2} d t & =O\left(T^{2-\uparrow \sigma}\right) \int_{1}^{T}|\zeta(1-\sigma-i t, K)|^{2} d t \\
& =O\left(T^{3-4 \sigma}\right)
\end{aligned}
$$

Hence

$$
\int_{\frac{T}{2}}^{T} \frac{|\zeta(\sigma+i t, K)|^{2}}{|\sigma+i t|^{2}} d t=O\left(T^{-\eta}\right)
$$

provided that $\sigma>\frac{1}{4}+\varepsilon$. It then follows by a simple argument that

$$
\int_{T}^{\infty} \frac{|\zeta(\sigma+i t, K)|^{2}}{|\sigma+i t|^{2}} d t \longrightarrow 0
$$

for $\sigma>\frac{1}{4}+\varepsilon$, and therefore that $\gamma \leqq \frac{1}{4}$ that is, that $\beta \leqq \frac{1}{4}$.

\section{REFERENCES}

1. G. H. Hardy, The average order of the arithmetical functions $P(x)$ and $\Delta(x)$, Proc. London Math. Soc. Series 2, 15 (1916), 192-213.

2. - A. Ingham, and G. Polya, Theorem concerning mean values of analytic functions, Proc. Royal Soc A, 113 (1926) 542-569.

3. Landau, Einfuhrung in die elementare und analyische Theorie der algebrischen Zahlen und der Ideale, (Göttingen 1917).

4. Z. Suetuna, On the product of L-functiom, Japanese J. Math. 2, (1925), 1937

5. E. C. Titchmarsh, On divisor problems, Quarterly J. Math. (Oxford) 9. (1938) 216-220.

6. - The theory of the Riemann zeta function, (Oxford, 1951).

7. .... Introduction to the thoery of Fourier integrals, (Oxford, 1931).

8. H. Weber, Lehrbuch der Algebra 2, (Braunschweig, 1908). 



\section{PACIFIC JOURNAL OF MATHEMATICS}

\section{EDITORS}

David Gilbarg

Stanford University

Stanford, California

R. A. Beaumont

University of Washington

Seattle 5 , Washington
A. L. Whiteman

University of Southern California Los Angeles 7, California

E. G. Straus

University of California

Los Angeles 24, California

\section{ASSOCIATE EDITORS}
E. F. BECKENBACH
C. E. BURGESS
M. HALL
E. HEWITT
A. HORN
V. GANAPATHY IYER
R. D. JAMES
M. S. KNEBELMAN

L. NACHBIN

I. NIVEN

T. G. OSTROM

H. L. ROYDEN
M. M. SCHIFFEI

G. SZEKERES

F. WOLF

K. YOSIDA

\section{SUPPORTING INSTITUTIONS}

UNIVERSITY OF BRITISH COLUMBIA

CALIFORNIA INSTITUTE OF TECHNOLOGY

UNIVERSITY OF CALIFORNIA

MONTANA STATE UNIVERSITY

UNIVERSITY OF NEVADA

OREGON STATE COLLEGE

UNIVERSITY OF OREGON

OSAKA UNIVERSITY

UNIVERSITY OF SOUTHERN CALIFORNIA
STANFORD UNIVERSITY

UNIVERSITY OF TOKYO

UNIVERSITY OF UTAH

WASHINGTON STATE COLLEGE

UNIVERSITY OF WASHINGTON

AMERICAN MATHEMATICAL SOCIETY

CALIFORNIA RESEARCH CORPORATION

HUGHES AIRCRAFT COMPANY

THE RAMO-WOOLDRIDGE CORPORATION

Printed in Japan by Kokusai Bunken Insatsusha

(International Academic Printing Co., Ltd.), Tokyo, Japan 


\section{Pacific Journal of Mathematics}

\section{Vol. 8, No. 1 \\ March, 1958}

Shimshon A. Amitsur, Commutative linear differential operators ......... 1

Masahiko Atsuji, Uniform continuity of continuous functions of metric

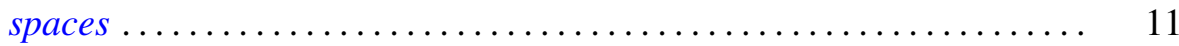

S. P. Avann, A numerical condition for modularity of a lattice . . . . . . . . . 17

Raymond G. D. Ayoub, A mean value theorem for quadratic fields........ 23

Errett Albert Bishop, Subalgebras of functions on a Riemann surface ..... . 29

Shaul Foguel, The relations between a spectral operator and its scalar

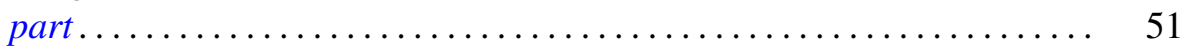

John Rolfe Isbell, Euclidean and weak uniformities ................. 67

Samuel Karlin and James L. McGregor, Many server queueing processes with Poisson input and exponential service times .............. 87

Paul Joseph Kelly and Ernst Gabor Straus, Curvature in Hilbert geometries....................................... 119

John W. Lamperti, Stationary measures for certain stochastic processes . . . 127

Richard Scott Pierce, Distributivity and the normal completion of Boolean

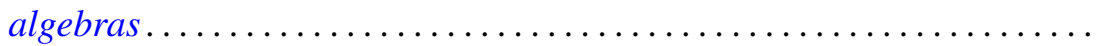

F. M. Ragab, Transcendental addition theorems for the hypergeometric

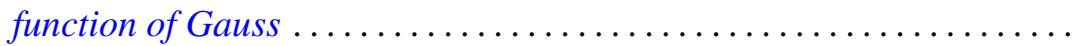

William T. Reid, Principal solutions of non-oscillatory self-adjoint linear differential systems ................................ 147

Maurice Sion, On general minimax theorems .................... 171

Chien Wenjen, On semi-normed ${ }^{*}$-algebras .................... 177 